\title{
Atualização em instabilidade patelar
}

\section{Update on Patellar Instability}

\author{
Nilson Roberto Severino ${ }^{1}$ Fabrício Roberto Severino ${ }^{1}$ Osmar Pedro Arbix de Camargo ${ }^{1}$ (1)

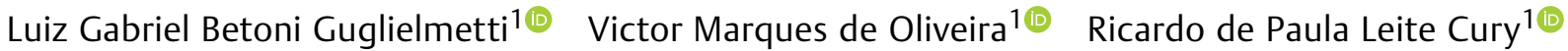

${ }^{1}$ Grupo de Joelho, Departamento de Ortopedia e Traumatologia, Faculdade de Ciências Médicas, Irmandade da Santa Casa de

Endereço para correspondência Nilson Roberto Severino, Misericórdia de São Paulo, Santa Casa de São Paulo, São Paulo, Brasil

\section{Resumo \\ Palavras-chave \\ - patela \\ - articulação patelofemoral \\ - instabilidade articular \\ - luxação patelar}

\section{Abstract}

\section{Keywords}

- patella

- patellofemoral joint

- joint instability

- patellar dislocation
A instabilidade patelar é uma condição clínica multifatorial, que acomete um número expressivo de pacientes, ocorrendo devido a variações anatômicas, morfológicas da articulação e do alinhamento patelofemoral. O presente estudo de revisão e atualização da literatura teve como objetivos identificar e sumarizar os conceitos atuais sobre instabilidade patelar em relação aos fatores de risco associados, os critérios diagnósticos e os benefícios e riscos dos tratamentos conservador e cirúrgico. Para tanto, foi realizado um levantamento nas bases de dados eletrônicas MEDLINE (via Pubmed), LILACS e Cochrane Library. Conclui-se que o diagnóstico preciso depende da avaliação clínica detalhada, incluindo o histórico e possíveis fatores de risco individuais, além de exames de imagem. O tratamento inicial da instabilidade patelar é ainda controverso, e requer a combinação de intervenções conservadoras e cirúrgicas, levando em consideração tanto os tecidos moles quanto as estruturas ósseas, sendo estas últimas a razão mais comum para a escolha do tratamento cirúrgico, principalmente instabilidade patelar lateral.

Patellar instability is a multifactorial clinical condition that affects a significant number of patients and occurs due to morphological variations of the joint and patellofemoral alignment. The present literature review study aimed to identify and summarize current concepts on patellar instability, in relation to associated risk factors, diagnostic criteria, and the benefits and risks of conservative and surgical treatments. For this purpose, a search was conducted in the following electronic databases: MEDLINE (via Pubmed), LILACS and Cochrane Library. It is concluded that the accurate diagnosis depends on the detailed clinical evaluation, including the history and possible individual risk factors, as well as imaging exams. The initial treatment of patellar instability is still controversial, and requires the combination of conservative and surgical interventions, taking into consideration both soft tissues and bone structures, the latter being the most common reason for choosing surgical treatment, especially lateral patellar instability. recebido

10 de Setembro de 2019

aceito

17 de Março de 2020

Publicado online

Novembro 2, 2020
DOI https://doi.org/

$10.1055 / \mathrm{s}-0040-1713389$

ISSN 0102-3616. (c) 2020. Sociedade Brasileira de Ortopedia e Traumatologia. All rights reserved.

This is an open access article published by Thieme under the terms of the Creative Commons Attribution-NonDerivative-NonCommercial-License, permitting copying and reproduction so long as the original work is given appropriate credit. Contents may not be used for commercial purposes, or adapted, remixed, transformed or built upon. (https://creativecommons.org/ licenses/by-nc-nd/4.0/)

Thieme Revinter Publicações Ltda., Rua do Matoso 170, Rio de Janeiro, RJ, CEP 20270-135, Brazil 


\section{Introdução}

A instabilidade patelar é uma condição clínica comum e incapacitante que afeta principalmente indivíduos jovens. Fatores anatômicos como displasia da tróclea, morfologia patelar, localização da tuberosidade tibial e ruptura dos tecidos moles têm sido associados à luxação primária e à instabilidade secundária recorrente. ${ }^{1}$ A luxação patelofemoral é uma lesão incapacitante que representa 2 a 3\% de todas as lesões traumáticas do joelho, e afeta de 5 a 43 casos a cada 100.000 jovens e adolescentes ao ano. ${ }^{2-4}$ Esta condição pode causar morbidade significativa, uma vez que está associada às altas taxas de recorrência, lesões e fraturas da cartilagem patelofemoral, lesão dos tecidos moles adjacentes, que resultam em dor, diminuição da função e possível desenvolvimento de artrose patelofemoral. ${ }^{2,5}$

A instabilidade da articulação patelofemoral é multifatorial, a presença de fatores predisponentes como a anteversão femoral, torção tibial externa, torção femoral interna, geno valgo, displasia patelar, displasia troclear, patela alta, atrofia do vasto medial oblíquo, pé plano e hiperfrouxidão generalizada, podem influenciar a ocorrência de luxações recorrentes. ${ }^{6,7}$

As luxações patelares são principalmente laterais e o principal mecanismo de lesão durante a atividade física é a rotação interna do fêmur com o pé plantado, componente valgo e um deslocamento lateral súbito da patela, ou um impacto direto que desloca a patela da articulação. Contudo, também podem ocorrer após traumas de baixa energia em pessoas com fatores predisponentes. ${ }^{8,9}$ Cerca de $93 \%$ das luxações traumáticas da patela ocorrem durante a flexão com o movimento em valgo do joelho sem contato direto, enquanto as luxações mediais são exclusivamente traumáticas ou iatrogênicas. ${ }^{9}$

O quadro clínico é composto por queixas de sensação de falseio, dor intensa e derrame secundário. Segundo Sillanpää et al., ${ }^{9}$ em 2008, condições como hemartrose, fratura da faceta medial e lesão do ligamento patelofemoral medial (LPFM) ocorrem em quase todos os pacientes após luxação traumática da patela; além disso, a fratura osteocondral pode ser observada em $25 \%$ dos casos.

O tratamento da luxação patelar primária aguda visa reduzir o risco de recidiva ou subluxação dolorosa e prevenir a osteoartrose secundária. ${ }^{5}$ Historicamente, a escolha terapêutica inicial é conservadora para pacientes que tiveram um episódio de luxação aguda, com exceção dos casos de lesões osteocondrais associadas ou fraturas, onde o tratamento cirúrgico precoce é indicado. ${ }^{10}$ De acordo com os estudos da história natural da doença, 50\% a 70\% dos pacientes apresentam-se livres de luxações recorrentes após o tratamento conservador. ${ }^{11-14}$ Por outro lado, algumas publicações recentes sugerem altas taxas de instabilidade recorrente tanto para pacientes operados quanto não operados. ${ }^{2,15}$

As consequências da luxação patelar aguda primária no longo prazo incluem além de luxações recorrentes, instabilidade patelar, lesão da cartilagem, dor, limitação das atividades da vida diária e osteoartrose patelofemoral secundária, com risco de recidiva da luxação aumentado em seis vezes nos pacientes com histórico de luxação patelar contralateral. Nos casos onde ocorrerem $\geq 2$ episódios de luxação, o risco de recidiva é em torno de $50 \%$ se houve lesão do LPFM. $^{9,16}$ Entretanto, as taxas de recorrência e sintomas residuais de instabilidade após o tratamento conservador levam consequentemente à indicação cirúrgica. Apesar das altas taxas de incidência da instabilidade patelofemoral, o manejo dos pacientes com esta condição é complexo e permanece variável na literatura. ${ }^{2,8}$ Assim, o presente estudo de revisão e atualização da literatura teve como objetivos identificar e sumarizar os conceitos atuais sobre instabilidade patelar, em relação aos fatores de risco associados, os critérios diagnósticos e os benefícios e riscos dos tratamentos conservador e cirúrgico. Foi realizado um levantamento nas bases de dados eletrônicas MEDLINE (via Pubmed), LILACS e Cochrane Library, e incluídos apenas estudos com boa qualidade metodológica e alto nível de evidência como revisões sistemáticas, ensaios clínicos randomizados e estudos observacionais prospectivos. As palavras-chave (MeSH terms) utilizadas nas estratégias foram Patella OR "Patellar Dislocation”, adaptados para cada base de dados pesquisada.

\section{Fatores de Risco}

Estudos relatam que $>60 \%$ das luxações da patela ocorrem durante a prática de atividade física. Estima-se que as mulheres apresentam risco aproximado $33 \%$ maior do que os homens, ${ }^{3,17,18}$ apesar de alguns estudos não encontrarem diferença entre os gêneros para a ocorrência de luxação primária. ${ }^{5,18,19}$ A idade é outro fator de risco relevante, pois a maioria das luxações agudas da patela ocorre em adolescentes e adultos jovens, principalmente entre 10 e 17 anos de idade. ${ }^{3,17}$

Fatores de risco anatômicos e estruturais para instabilidade patelar foram identificados, tais como: patela alta, morfologia patelar anormal, displasia troclear, hipermobilidade patelar, variações da anatomia do LPFM, hiperfrouxidão ligamentar generalizada, hipoplasia do vasto medial oblíquo, aumento do ângulo $\mathrm{Q}$ aumento da anteversão femoral, alinhamento em valgo e rotação tibial externa. ${ }^{7,8,20}$

Dejour et al., ${ }^{21}$ em 1994, consideraram quatro fatores predisponentes à luxação: 1) displasia da tróclea; 2) inclinação lateral da patela, interpretada como displasia do vasto medial oblíquo; 3) patela alta e 4) lateralização da tuberosidade anterior da tíbia (TAT) (aumento do ângulo Q). Um estudo observacional prospectivo analisou a morfologia patelofemoral nas crianças esqueleticamente imaturas com e sem luxação primária da patela, e encontraram diferenças significativas nas medidas relacionadas à displasia troclear como maior ângulo do sulco e menor profundidade troclear $(<3 \mathrm{~mm}$ ), que resultam em aumento da altura condilar central. ${ }^{19}$ Outro estudo prospectivo ${ }^{18}$ analisou os mesmos fatores de risco em crianças e adultos e mostrou achados semelhantes, ou seja, a displasia troclear e a altura patelar excessiva foram os fatores mais comuns, sem diferenças em relação à maturidade esquelética. A combinação de dois ou mais fatores de risco associados está presente na maioria dos pacientes que evoluem para luxação primária da patela. ${ }^{3}$ Segundo Santos Netto et al., ${ }^{22}$ em 2012, a morfologia do LPFM varia com a distância interepicondilar e do côndilo lateral, e com a idade do paciente. 
A recorrência de luxação patelar foi investigada por estudos coorte prospectivos que mostraram incidência total acumulada de recidiva ipsilateral entre $36^{23}$ e $54 \%{ }^{24}$ após 15 a 20 anos, sendo significativamente maior em pacientes $<18$ anos na primeira luxação, presença de displasia da tróclea, patela alta e maior distância entre a tuberosidade da tíbia e o sulco troclear(TA-GT). A maioria dos eventos ocorreu dentro de 5 anos da lesão inicial. Ainda, aproximadamente metade dos pacientes que sofreram luxação lateral da patela apresentam sintomas e alterações radiográficas compatíveis com osteoartrose em média após 25 anos da lesão. ${ }^{25}$ Assim, falhas no tratamento da luxação patelar podem levar à instabilidade patelar recorrente, dor persistente no joelho e até mesmo osteoartrose patelofemoral. ${ }^{26}$

\section{Critérios Diagnósticos}

Embora o exame físico seja a principal ferramenta diagnóstica para instabilidade patelar, os exames de imagem são utilizados para auxiliar na tomada de decisão clínica e no diagnóstico diferencial, e incluem radiografias convencionais, ressonância nuclear magnética (RNM), tomografia computadorizada (TC) e ultrassonografia (US). 1,27 A avaliação radiológica é frequentemente o primeiro exame de imagem a ser solicitado, incluindo as incidências a) anteroposterior, para avaliação do alinhamento articular e simetria das estruturas ósseas; b) perfil absoluto em 30 graus de flexão, o qual permite a mensuração da altura patelar a partir da relação entre a patela e a tíbia utilizando índices como Insall-Salvatti, ${ }^{28}$ Blackburne-Peel ${ }^{29} \mathrm{e}$ Caton-Deschamps, ${ }^{30}$ além da medida do ângulo platôpatela $^{31}$; (c) axial, para avaliação do ângulo do sulco troclear e do formato e posicionamento (inclinação) da patela.

Uma revisão sistemática mostrou que a mensuração radiográfica da altura patelar por meio dos métodos de Insall-Salvatti e Caton-Deschamps, e de ressonância magnética (RM) pelo método Insall-Salvatti, apresentaram boa validade, com diferença significativa entre amostras de pacientes saudáveis e com instabilidade patelar $(\mathrm{p}<0,0001)$. A medida da distância TA-GT avaliada pela TC também demonstrou boa validade intra- e interexaminador. As medidas de inclinação patelar (tilt patelar) e dos ângulos do sulco troclear e patelar lateral também apresentaram boa validade de discriminação entre os indivíduos com instabilidade patelar e o grupo controle $(\mathrm{p}<0,0001)$. Para as medidas do ângulo de congruência e altura dos côndilos lateral e medial, houve baixa validade além da heterogeneidade substancial entre os estudos analisados. Por fim, não há evidências suficientes para determinar a confiabilidade e validade da altura patelar utilizando o método de Blackburne-Peel, o ângulo de congruência pela RNM, o deslocamento patelar lateral pela RNM, a anteversão femoral pela TC, a profundidade da tróclea, o sinal do cruzamento e a classificação patelar de Wiberg. Houve baixa confiabilidade para a avaliação da displasia troclear e do ângulo do sulco pelo US. ${ }^{1,32}$

\section{Tratamento}

\section{Tratamento conservador}

O tratamento da instabilidade e luxação patelar permanece um desafio para os cirurgiões devido à complexidade dos procedimentos e os resultados insatisfatórios. Historicamente, o tratamento conservador (farmacológico, fisioterapia e imobilização) foi considerado como primeira opção para as luxações patelares primárias, exceto na presença de fraturas osteocondrais ${ }^{15}$ e avulsão do LPFM. $^{33}$ Entretanto, ainda existem controvérsias na literatura a respeito da indicação para o tratamento conservador ou cirúrgico. Alguns estudos encontraram altas taxas de recidiva e sintomas residuais de instabilidade associados ao tratamento conservador (até 44\%), ${ }^{13,14,26,34}$ e por esta razão, o tratamento cirúrgico tem sido preconizado, porém também apresenta taxas elevadas de recorrência (10 a 35\%). ${ }^{26}$

Uma revisão sistemática Cochrane ${ }^{4}$ publicada em 2015 mostrou que embora haja alguma evidência para apoiar o tratamento cirúrgico na luxação patelar primária, a qualidade da evidência é muito baixa devido ao alto risco de viés e imprecisão nas estimativas de efeito dos estudos incluídos. Portanto, novos ensaios clínicos randomizados de melhor qualidade metodológica foram recomendados. A - Tabela 1- ${ }^{4,8,26,35,36}$ mostra os resultados das revisões sistemáticas com metanálise comparando o tratamento conservador ao cirúrgico para luxação primária da patela.

De modo geral, os objetivos do tratamento conservador após a luxação primária da patela são reduzir o inchaço, fortalecer a musculatura do joelho e melhorar a amplitude de movimento articular. ${ }^{33}$ A fisioterapia para pacientes com instabilidade patelar deve centrar-se no fortalecimento de cadeia cinética fechada da musculatura do quadríceps e glúteo, e treinamento sensório-motor. Exercícios de cadeia cinética fechada envolvendo a musculatura glútea tendem a forçar a rotação externa do fêmur e, consequentemente, diminuem o ângulo $Q$ durante o ciclo da marcha. O fortalecimento da musculatura do quadríceps com ênfase no vasto medial oblíquo (VMO) auxilia no posicionamento adequado da patela no sulco troclear. . $^{33,34,37,38}$ o período de imobilização após episódio de luxação varia na literatura entre 2 a 6 semanas. $^{8}$

\section{Tratamento cirúrgico}

A seleção do procedimento cirúrgico apropriado depende da fisiopatologia individual da instabilidade patelar que, em sua maioria, é de natureza anatômica. Por esta razão, as técnicas cirúrgicas têm como objetivo restaurar a anatomia normal, existindo associação entre as luxações laterais da patela e as lesões dos tecidos moles mediais. ${ }^{15}$ As indicações para o tratamento cirúrgico também estão relacionadas à dor e à função do paciente. Em muitos casos, os pacientes apresentam sintomas mínimos ao repouso, mas limitam significativamente suas atividades funcionais devido à apreensão e instabilidade. Portanto, o risco de recorrência é um elemento importante a ser considerado no tratamento. ${ }^{6} \mathrm{O}$ objetivo do tratamento cirúrgico da instabilidade da patela é estabilizála, restaurar a cinemática normal e otimizar a transmissão das cargas através da articulação. As técnicas cirúrgicas incluem procedimentos ósseos, como a osteotomia da tuberosidade da tíbia, com medialização e/ou distalização e a trocleoplastia; intervenções nos tecidos moles, como a reconstrução do LPFM. $^{39,40}$ Técnicas artroscópicas e 
Tabela 1 Resultados das revisões sistemáticas com metanálise sobre tratamento conservador versus cirúrgico para luxação patelar primária

\begin{tabular}{|c|c|c|c|}
\hline Estudo/Ano & $\begin{array}{l}\text { Estudos incluídos e } \\
\text { participantes }\end{array}$ & $\begin{array}{l}\text { Qualidade dos estudos } \\
\text { incluídos (GRADE) }\end{array}$ & Resultados \\
\hline \multirow[t]{2}{*}{ Yang et al., ${ }^{35} 2019$} & \multirow{2}{*}{$\begin{array}{l}16 \text { ECRs/estudos } \\
\text { observacionais } \\
\mathrm{N}=918 \text { participantes } \\
23 \text { a } 36 \text { anos } \\
\text { Follow up: } 1 \text { a } 5 \text { anos }\end{array}$} & \multirow[t]{2}{*}{ Baixa } & $\begin{array}{l}\text { Diferença significativa a favor da cirurgia: } \\
\text { - Kujala: DMP } 0,79[0,30 \text { a } 1,28] \\
\text { - Recidiva: OR } 0,44[0,30 \text { a } 0,63]\end{array}$ \\
\hline & & & $\begin{array}{l}\text { Não houve diferença significativa entre os grupos: } \\
\text { - Dor (EAV): DMP } 0,84[0,36 \text { a } 9,03]\end{array}$ \\
\hline Lee et al., ${ }^{26} 2018$ & $\begin{array}{l}4 \text { ECRs } \\
\mathrm{N}=275 \text { participantes } \\
23 \text { a } 36 \text { anos } \\
\text { Follow up: } 1 \text { a } 5 \text { anos }\end{array}$ & Moderada a baixa & $\begin{array}{l}\text { Não houve diferença significativa entre os grupos: } \\
\text { - Recidiva: RR } 1,33[0,89 \text { a } 2,00] \\
\text { - Kujala: DM } 1,76[-2,02 \text { a } 5,54] \\
\text { - Instabilidade: RR } 1,11[0,89 \text { a } 1,40] \\
\text { - Dor: DM - } 0,39[-6,97 \text { a } 6,20] \\
\text { - Tegner: DM } 0,63[-0,32 \text { a } 1,58]\end{array}$ \\
\hline Longo et al., ${ }^{36} 2017$ & $\begin{array}{l}17 \text { ECRs/estudos } \\
\text { observacionais } \\
\mathrm{N}=2.086 \text { ( } 2.134 \text { joelhos }) \\
\text { Média } 20,3 \text { anos } \\
\text { Follow up: média } 5 \text { anos }\end{array}$ & NA & $\begin{array}{l}\text { Diferença significativa a favor da cirurgia: } \\
\text { - Recidiva: OR } 0,54[0,40 \text { a } 0,70] \\
\text { (36,4\% conservador x } 25 \% \text { cirúrgico) }\end{array}$ \\
\hline \multirow[t]{2}{*}{$\begin{array}{l}\text { Saccomano et al., } \\
2016\end{array}$} & \multirow{2}{*}{$\begin{array}{l}9 \text { ECRs } \\
\mathrm{N}=430 \text { joelhos } \\
13 \text { a } 36 \text { anos } \\
\text { Follow up: } 2 \text { a } 14 \text { anos }\end{array}$} & \multirow[t]{2}{*}{ Moderada a baixa } & $\begin{array}{l}\text { Diferença significativa a favor da cirurgia: } \\
\text { - Recidiva: RR } 0,62[0,39 \text { a } 0,98] \\
\text { - Dor: DMP - } 0,32[-0,61 \text { a }-0,03]\end{array}$ \\
\hline & & & $\begin{array}{l}\text { Não houve diferença significativa entre os grupos: } \\
\text { - Kujala: DMP } 0,74[0,08 \text { a } 1,40] \\
\text { - Lysholm: DMP - 0,10 [- } 0,45 \text { a } 0,26] \\
\text { - Tegner: DMP - 0,61 [- } 1.25 \text { a } 0.02]\end{array}$ \\
\hline \multirow[t]{2}{*}{ Smith et al., ${ }^{4} 2015$} & \multirow[t]{2}{*}{$\begin{array}{l}6 \text { ECRs } \\
\mathrm{N}=344 \text { participantes } \\
19 \text { a } 27 \text { anos } \\
\text { Follow up: até } 2 \text { anos }\end{array}$} & \multirow[t]{2}{*}{ Muito baixa } & $\begin{array}{l}\text { Diferença significativa a favor da cirurgia } \\
\text { (após } 2 \text { a } 5 \text { anos): } \\
\text { - Recidiva: RR } 0,53[0,33 \text { a } 0,87] \\
\text { - Kujala: DM } 13,93[5,33 \text { a } 22,53] \\
\text { - Instabilidade: RR } 0,44[0,27 \text { a } 0,72]\end{array}$ \\
\hline & & & $\begin{array}{l}\text { Não houve diferença significativa entre os grupos: } \\
\text { - Recidiva: } \\
\text { após } 6 \text { a } 9 \text { anos: RR } 0,67[-0,42 \text { a } 1,08] \\
\text { após } 14 \text { anos RR } 0,93[-0,67 \text { a } 1,30] \\
\text { - Kujala: } \\
\text { após } 6 \text { a } 9 \text { anos: RR - } 0,35[-10,61 \text { a } 4,11] \\
\text { após } 14 \text { anos RR - } 1,00[-8,60 \text { a } 6,60]\end{array}$ \\
\hline
\end{tabular}

Abreviações: DM, diferença de média; DMO, diferença de média padronizada; EAV, escala analógica visual de dor; ECR, ensaio cínico randomizado; GRADE, The Grading of Recommendations Assessment, Development and Evaluation; NA, não avaliado; OR, odds ratio; RR, risco relativo.

minimamente invasivas, como a plicatura medial e as liberações retinaculares, são tipicamente recomendadas para pacientes com mínimo desalinhamento ósseo ou displasia troclear, ou como adjuvantes para proporcionar equilíbrio adicional aos tecidos moles em pacientes submetidos a procedimentos de reparo ósseo. ${ }^{41}$ Entretanto, a liberação retinacular lateral artroscópica isolada pode resultar em aumento da mobilidade lateral da patela e instabilidade medial, e não é recomendada. ${ }^{6}$

\section{Reconstrução do ligamento patelofemoral medial}

Entre as técnicas cirúrgicas para a luxação patelar recorrente, a restauração da função do LPFM é um dos fatores mais importantes. O LPFM é o principal restritor à translação lateral da patela, e contribui com 50 a $60 \%$ da força de contenção medial contra a translação patelar lateral. Entretanto, ao comparar as técnicas de reconstrução do LPFM, a escolha do enxerto, o posicionamento deste enxerto ou a sua tensão, é difícil, dada a escassez de evidências clínicas de estudos comparativos. ${ }^{26,42}$ A reconstrução do LPFM é indicada para pacientes que apresentam lesão ou deficiência do LFPM e que apresentam instabilidade recorrente sem evidência de mau alinhamento ósseo. 0 diagnóstico artroscópico também pode ser realizado antes da reconstrução do LPFM para identificar quaisquer lesões osteocondrais ou corpos livres. ${ }^{43}$ As técnicas reconstrutivas do LFPM envolvem enxerto autólogo dos tendões flexores (grácil ou semitendinoso), passado através de um túnel ósseo patelar no fêmur ou fixado à patela com âncoras de sutura. 0 tendão do quadríceps também pode ser utilizado como enxerto para o LPFM. ${ }^{43}$ O ângulo de flexão do joelho durante a fixação do enxerto do LPFM varia de $20^{\circ}$ a $90^{\circ}$. A fixação do enxerto em ângulos menores e maiores de flexão do joelho, durante a reconstrução do LPFM, mostrou resultados satisfatórios relatados 
pelo paciente e baixas taxas de recidiva. ${ }^{44} \mathrm{~A}$ respeito da comparação entre o enxerto em banda única ou dupla banda, parece não haver diferenças quanto à melhora da função do joelho, taxa de recidiva e ocorrência de complicações. A técnica em banda simples pode apresentar maior risco de apreensão pós-operatória, e a dupla banda pode causar maior rigidez articular, porém não há evidências suficientes na literatura para apoiar ou refutar estes achados. ${ }^{45,46}$

A maioria dos estudos sobre a reconstrução do LPFM, isolada ou combinada a outras técnicas, mostram resultados positivos, com melhora da dor e da função física e baixa incidência de luxações recorrentes. Uma revisão sistemática ${ }^{47}$ analisou os efeitos desta cirurgia em pacientes esqueleticamente imaturos (média 13,2 anos de idade e 4,8 anos de pósoperatório, enxerto autólogo de flexores). Houve melhora significativa do questionário Kujala de 59,1 para 84,6 pontos antes e após a cirurgia. A taxa de complicações foi de $25 \%$, sendo $3,8 \%$ reluxação e $11,4 \%$ subluxação. A revisão sistemática de Lee et al., ${ }^{26}$ em 2018, comparou as técnicas de realinhamento medial e reconstrução do LPFM e observou diferença significativa a favor da reconstrução na função física avaliada pelos questionários Kujala (diferença de média [DM] 8,91 [- 14,05 a - 3,77]) e Lysholm (DM - 13,51 [- 21,35 a - 5,68]).

Uma abordagem cirúrgica combinada parece ser indicada para pacientes com aumento do sulco tubérculo-troclear da tíbia (TAS-GT) ou displasia troclear grave. A indicação para a reconstrução do LPFM combinada a um procedimento ósseo como a osteotomia é influenciada pelas alterações anatômicas individuais. ${ }^{39}$

\section{Osteotomia da tuberosidade anterior da tíbia}

A osteotomia da tuberosidade tibial é outra opção de tratamento para pacientes com instabilidade patelofemoral. Refere-se à alteração da posição da tuberosidade tibial, realinhamento do mecanismo extensor do joelho e correção do desalinhamento e da instabilidade. Este procedimento é indicado em pacientes com instabilidade recorrente, aumento da distância TA-GT, patela alta ou lesões osteocondrais de alto grau na articulação patelofemoral. ${ }^{33,40,43}$ Uma das técnicas mais comuns é a osteotomia de anteromedialização descrita por Fulkerson, ${ }^{48}$ que envolve a transferência da tuberosidade tibial anteromedialmente, e é indicada para pacientes com aumento da distância TA-GT, patela alta, ou lesões osteocondrais. A medialização da TAT, também conhecida como procedimento de Elmslie-Trillat, é uma opção para tratar o mau alinhamento patelar em pacientes sem patela alta. ${ }^{43}$ Os resultados após a transferência da TAT têm mostrado bons resultados. Uma revisão sistemática analisou 38 estudos com um total de 1.182 cirurgias, e relatou uma taxa de recorrência da luxação de $7 \%{ }^{49}$

\section{Trocleoplastia}

A instabilidade patelar com displasia troclear grave é a principal indicação para a trocleoplastia, uma técnica cirúrgica capaz de corrigir e remodelar a arquitetura óssea da tróclea femoral. ${ }^{50}$ Indicações específicas para a trocleoplastia incluem pacientes com mau alinhamento patelar extremo (identificado pelo sinal "J" ao exame clínico e distância TA-
GT $>10 \mathrm{~mm}$ ), morfologia troclear anormal, na presença de cartilagem articular normal ou quase normal. ${ }^{15,51}$

Diferentes técnicas para correção cirúrgica da displasia troclear foram descritas: 1) elevação da faceta lateral; 2) aprofundamento do sulco com ou sem remoção troclear subcondral, seguido pela fixação da cartilagem troclear corrigida; e 3) ressecção de um retalho cartilaginoso subcondral, remodelamento da tróclea e refixação da cartilagem. ${ }^{50,52}$

A trocleoplastia é contraindicada para pacientes com placa epifisária aberta ou com diagnóstico de artrose patelofemoral difusa. As complicações associadas à trocleoplastia incluem artrite como resultado de dano à cartilagem da tróclea, e artrofibrose. ${ }^{53}$ Dadas as exigências técnicas e potenciais complicações, os desfechos clínicos após a trocleoplastia apresentam índices de satisfação entre 67 e 95\%. ${ }^{15,52}$

Segundo Banke et al., ${ }^{52}$ a trocleoplastia combinada à reconstrução do LPFM tem mostrado bons resultados para o tratamento da instabilidade patelar crônica. Este conceito de tratamento associado é uma opção segura, não apenas como terapia de resgate, mas também como procedimento primário em relação ao tratamento de pacientes com alterações anatômicas importantes, como a displasia troclear grave. A reconstrução isolada do LPFM, para estes casos, segundo os autores, pode levar a $46 \%$ de luxação recidivante.

\section{Deformidades angulares}

Segundo Zhang et al., ${ }^{54}$ as deformidades femorais, tais como valgo ou rotativas, podem levar à luxação patelar e, algumas vezes, tem que ser corrigidas. A osteotomia varizante distal e a osteotomia desrrotativa externa do fêmur podem, muitas vezes, evitar novas luxações da patela, sem a necessidade de outras associações corretivas. Segundo os autores, a rotação interna do fêmur deve ser excessiva para que se faça a osteotomia, citando 4 casos com $30^{\circ}, 45^{\circ}$ e $60^{\circ}$ de torção interna do fêmur, nos quais realizaram osteotomia desrrotativa externa e não mais ocorreram luxações.

\section{Conclusão}

As evidências científicas disponíveis a respeito do tratamento da instabilidade patelar ainda são controversas e de qualidade metodológica baixa a moderada. Contudo, a atualização a respeito das estratégias de tratamento e diagnóstico, e do conhecimento dos fatores de risco desta lesão, são importantes para auxiliar a tomada de decisão do cirurgião. É fundamental compreender a importância da técnica cirúrgica no que se refere às alterações anatômicas individuais envolvidas. Estudos futuros com maior rigor metodológico ainda são necessários para abordar os tópicos que permanecem incertos.

Conflito de Interesses

Os autores declaram não haver conflito de interesses.

\section{Referências}

1 Smith TO, Davies L, Toms AP, Hing CB, Donell ST. The reliability and validity of radiological assessment for patellar instability. A 
systematic review and meta-analysis. Skeletal Radiol 2011;40 (04):399-414

2 Liu JN, Steinhaus ME, Kalbian IL, et al. Patellar Instability Management: A Survey of the International Patellofemoral Study Group. Am J Sports Med 2018;46(13):3299-3306

3 Fithian DC, Paxton EW, Stone ML, et al. Epidemiology and natural history of acute patellar dislocation. Am J Sports Med 2004;32 (05):1114-1121

4 Smith TO, Donell S, Song F, Hing CB. Surgical versus non-surgical interventions for treating patellar dislocation. Cochrane Database Syst Rev 2015;(02):CD008106

5 Arendt EA, Fithian DC, Cohen E. Current concepts of lateral patella dislocation. Clin Sports Med 2002;21(03):499-519

6 Koh JL, Stewart C. Patellar instability. Clin Sports Med 2014;33 (03):461-476

7 Franco BAFM, Sadigursky D, Daltro GC. Patellar position in patients with patellofemoral syndrome as characterized by anatomo-radiographic study. Rev Bras Ortop 2018;53(04):410-414

8 Saccomanno MF, Sircana G, Fodale M, Donati F, Milano G. Surgical versus conservative treatment of primary patellar dislocation. A systematic review and meta-analysis. Int Orthop 2016;40(11): 2277-2287

9 Sillanpää P, Mattila VM, Iivonen T, Visuri T, Pihlajamäki H. Incidence and risk factors of acute traumatic primary patellar dislocation. Med Sci Sports Exerc 2008;40(04):606-611

10 Panni AS, Vasso M, Cerciello S. Acute patellar dislocation. What to do? Knee Surg Sports Traumatol Arthrosc 2013;21(02):275-278

11 Cofield RH, Bryan RS. Acute dislocation of the patella: results of conservative treatment. J Trauma 1977;17(07):526-531

12 Hawkins RJ, Bell RH, Anisette G. Acute patellar dislocations. The natural history. Am J Sports Med 1986;14(02):117-120

13 Mäenpää $H$, Huhtala $H$, Lehto MU. Recurrence after patellar dislocation. Redislocation in 37/75 patients followed for 6-24 years. Acta Orthop Scand 1997;68(05):424-426

14 Mäenpää H, Lehto MU. Patellar dislocation. The long-term results of nonoperative management in 100 patients. Am J Sports Med 1997;25(02):213-217

15 Weber AE, Nathani A, Dines JS, et al. An Algorithmic Approach to the Management of Recurrent Lateral Patellar Dislocation. J Bone Joint Surg Am 2016;98(05):417-427

16 Duthon VB. Acute traumatic patellar dislocation. Orthop Traumatol Surg Res 2015;101(01):S59-S67

17 Seitlinger G, Ladenhauf HN, Wierer G. What is the chance that a patella dislocation will happen a second time: update on the natural history of a first time patella dislocation in the adolescent. Curr Opin Pediatr 2018;30(01):65-70

18 Arendt EA, England K, Agel J, Tompkins MA. An analysis of knee anatomic imaging factors associated with primary lateral patellar dislocations. Knee Surg Sports Traumatol Arthrosc 2017;25(10): 3099-3107

19 Askenberger M, Janarv PM, Finnbogason T, Arendt EA. Morphology and Anatomic Patellar Instability Risk Factors in First-Time Traumatic Lateral Patellar Dislocations: A Prospective Magnetic Resonance Imaging Study in Skeletally Immature Children. Am J Sports Med 2017;45(01):50-58

20 Balcarek P, Oberthür S, Hopfensitz S, et al. Which patellae are likely to redislocate? Knee Surg Sports Traumatol Arthrosc 2014; 22(10):2308-2314

21 Dejour H, Walch G, Nove-Josserand L, Guier C. Factors of patellar instability: an anatomic radiographic study. Knee Surg Sports Traumatol Arthrosc 1994;2(01):19-26

22 Santos Netto A, Brito MB, Severino FR, et al. Estudo da articulação patelofemoral por ressonância magnética: a variação da morfologia do ligamento patelofemoral medial. Rev Bras Ortop 2012;47 (02):204-209

23 Christensen TC, Sanders TL, Pareek A, Mohan R, Dahm DL, Krych AJ. Risk Factors and Time to Recurrent Ipsilateral and Contralateral Patellar Dislocations. Am J Sports Med 2017;45(09):2105-2110
24 Sanders TL, Pareek A, Hewett TE, Stuart MJ, Dahm DL, Krych AJ. High rate of recurrent patellar dislocation in skeletally immature patients: a long-term population-based study. Knee Surg Sports Traumatol Arthrosc 2018;26(04):1037-1043

25 Sanders TL, Pareek A, Johnson NR, Stuart MJ, Dahm DL, Krych AJ. Patellofemoral Arthritis After Lateral Patellar Dislocation: A Matched Population-Based Analysis. Am J Sports Med 2017;45(05): 1012-1017

26 Lee DY, Park YJ, Song SY, Hwang SC, Park JS, Kang DG. Which Technique Is Better for Treating Patellar Dislocation? A Systematic Review and Meta-analysis. Arthroscopy 2018;34(11): 3082-3093.e1

27 Souza PME, Gabetto MSS, Serrão MG, Vieira LAM, Oliveira DC. Patellofemoral Instability: Evaluation by Magnetic Resonance Imaging. Rev Bras Ortop 2013;48(02):159-164

28 Insall J, Salvati E. Patella position in the normal knee joint Radiology 1971;101(01):101-104

29 Blackburne JS, Peel TE. A new method of measuring patellar height. J Bone Joint Surg Br 1977;59(02):241-242

30 Caton J. [Method of measuring the height of the patella]. Acta Orthop Belg 1989;55(03):385-386

31 Portner O, Pakzad H. The evaluation of patellar height: a simple method. J Bone Joint Surg Am 2011;93(01):73-80

32 Haj-Mirzaian A, Thawait GK, Tanaka MJ, Demehri S. Diagnosis and Characterization of Patellofemoral Instability: Review of Available Imaging Modalities. Sports Med Arthrosc Rev 2017; 25(02):64-71

33 Koh JL, Stewart C. Patellar instability. Orthop Clin North Am 2015; 46(01):147-157

34 Lewallen LW, McIntosh AL, Dahm DL. Predictors of recurrent instability after acute patellofemoral dislocation in pediatric and adolescent patients. Am J Sports Med 2013;41(03):575-581

35 Yang F, Guo W, Wang $Q$ et al. Surgical versus nonsurgical treatment of primary acute patellar dislocation: A systematic review and meta-analysis. Medicine (Baltimore) 2019;98(29):e16338

36 Longo UG, Ciuffreda M, Locher J, Berton A, Salvatore G, Denaro V. Treatment of Primary Acute Patellar Dislocation: Systematic Review and Quantitative Synthesis of the Literature. Clin J Sport Med 2017;27(06):511-523

37 Dolak KL, Silkman C, Medina McKeon J, Hosey RG, Lattermann C, Uhl TL. Hip strengthening prior to functional exercises reduces pain sooner than quadriceps strengthening in females with patellofemoral pain syndrome: a randomized clinical trial. J Orthop Sports Phys Ther 2011;41(08):560-570

38 Cowan SM, Bennell KL, Crossley KM, Hodges PW, McConnell J. Physical therapy alters recruitment of the vasti in patellofemoral pain syndrome. Med Sci Sports Exerc 2002;34(12): 1879-1885

39 Longo UG, Berton A, Salvatore G, et al. Medial Patellofemoral Ligament Reconstruction Combined With Bony Procedures for Patellar Instability: Current Indications, Outcomes, and Complications. Arthroscopy 2016;32(07):1421-1427

40 Dejour D, Le Coultre B. Osteotomies in Patello-Femoral Instabilities. Sports Med Arthrosc Rev 2018;26(01):8-15

41 Fonseca LPRMD, Kawatake EH, Pochini AC. Lateral patellar retinacular release: changes over the last ten years. Rev Bras Ortop 2017;52(04):442-449

42 Baumann CA, Pratte EL, Sherman SL, Arendt EA, Hinckel BB. Reconstruction of the medial patellotibial ligament results in favorable clinical outcomes: a systematic review. Knee Surg Sports Traumatol Arthrosc 2018;26(10):2920-2933

43 Buchanan G, Torres L, Czarkowski B, Giangarra CE. current concepts in the treatment of gross patellofemoral instability. Int J Sports Phys Ther 2016;11(06):867-876

44 Patel NK, de Sa D, Vaswani R, Kay J, Musahl V, Lesniak BP. Knee Flexion Angle During Graft Fixation for Medial Patellofemoral Ligament Reconstruction: A Systematic Review of Outcomes and Complications. Arthroscopy 2019;35(06):1893-1904 
45 Kang H, Zheng R, Dai Y, Lu J, Wang F. Single- and double-bundle medial patellofemoral ligament reconstruction procedures result in similar recurrent dislocation rates and improvements in knee function: a systematic review. Knee Surg Sports Traumatol Arthrosc 2019;27(03):827-836

46 Sadigursky D, de Melo Laranjeira SM, Nunes M, Caneiro RJ, Colavolpe PO. Reconstruction of the medial patellofemoral ligament by means of the anatomical double-bundle technique using metal anchors. Rev Bras Ortop 2016;51(03):290-297

47 Shamrock AG, Day MA, Duchman KR, Glass N, Westermann RW. Medial Patellofemoral Ligament Reconstruction in Skeletally Immature Patients: A Systematic Review and Meta-analysis. Orthop J Sports Med 2019;7(07):2325967119855023

48 Fulkerson JP. Anteromedialization of the tibial tuberosity for patellofemoral malalignment. Clin Orthop Relat Res 1983;(177): 176-181

49 Longo UG, Rizzello G, Ciuffreda M, et al. Elmslie-Trillat, Maquet, Fulkerson, Roux Goldthwait, and Other Distal Realignment Procedures for the Management of Patellar Dislocation: Systematic
Review and Quantitative Synthesis of the Literature. Arthroscopy 2016;32(05):929-943

50 Nolan JE III, Schottel PC, Endres NK. Trochleoplasty: Indications and Technique. Curr Rev Musculoskelet Med 2018;11(02): 231-240

51 Carstensen SE, Menzer HM, Diduch DR. Patellar Instability: When is Trochleoplasty Necessary? Sports Med Arthrosc Rev 2017;25 (02):92-99

52 Banke IJ, Kohn LM, Meidinger G, et al. Combined trochleoplasty and MPFL reconstruction for treatment of chronic patellofemoral instability: a prospective minimum 2-year follow-up study. Knee Surg Sports Traumatol Arthrosc 2014; 22(11):2591-2598

53 LaPrade RF, Cram TR, James EW, Rasmussen MT. Trochlear dysplasia and the role of trochleoplasty. Clin Sports Med 2014; 33(03):531-545

54 Zhang X, Attenello J, Safran MR, Lowenberg DW. Congenital internal rotation deformity of the distal femur presenting as patellofemoral instability and pain. J ISAKOS 2019;4:93-97 